\title{
Nachtrag zu dem Bericht über den 2. allgemeinen ärztlichen Kongreß für Psychotherapie in Bad Nauheim.
}

\author{
(Eingegangen am 24. Oktober 192\%.)
}

\begin{abstract}
Roemer-Karlsruhe: Psychotherapeutische Gesichtspunkte in der offenen Geisteskrankentürsorge.

1. Die offene Geisteskrankenfürsorge bedeutet das folgerichtige Endglied einer zunehmend freiheitlicheren Entwicklung der Irrenpflege, die seit der Mitte des vorigen Jahrhunderts von der Anwendung des mechanischen Zwanges zur Beschäftigungs-, Bett- und Dauerbad behandlung, von den kasernenartigen Trakten mit Zellkorridoren zu den Pavillon-Anstalten mit Offentürsystem, Werkstätten, landwirtschaftlichen Kolonien und Familienpflege fortgeschritten ist. Die offene Geisteskrankenfürsorge, die den Kranken im Verband seines Familien und Erwerbslebens betreut, ist die schonendste und freieste aller psychiatrischen Fürsorgeformen, sei es, daß sie die Anstaltspflege fortsetzt und zu Ende führt, sei es, daß sie diese ersetzt und erspart.
\end{abstract}

2. Während die anatomisch-neurologische Forschung in der wissenschaftlichen Psychiatrie zusammen mit der anschließenden ätiologischklinischen Richtung in der Theorie eine Überbewertung des ,,schicksalsmäßig verlaufenden" endogenen biologischen Geschehens und in der Praxis einen therapeutischen Nihilismus zur Folge hatte, hat die neuerdings vorherrschende psychologische und psychopathologische Betrachtungsweise die große Reichweite des psychogenen Faktors in der Symptomatologie der Psychosen und Psychopathien und damit die Gefährdung der Anstaltsinsassen, insbesondere der zahlreichen Schizophrenen durch die in jedem Fall künstlichen Verhältnisse des Anstalts. milieus entdeckt. Die unter diesem Gesichtspunkt zur Abkürzung des Anstaltsaufenthaltes und zur Bekämpfung des Hospitalismus zu fordernde psychotherapeutische Frühentlassung findet in der nachgehenden Fürsorge eine wesentliche technische Erleichterung, ja in vielen Fällen die unerläßliche Voraussetzung für die soziale Wiedereingliederung 
des zu entlassenden Kranken. Außerdem ist die offene Fürsorge imstande, manche Unterbringung in der geschlossenen Anstalt hinauszuschieben oder ganz zu ersparen.

3. Die offene Geisteskrankenfürsorge hat wie die übrigen Zweige der Gesundheitsfürsorge (Säuglings-, Tuberkulose-, Krüppel-, Geschlechtskranken- usw. Fürsorge) die Aufgabe, den einzelnen Kranken bzw. Krankheitsgefährdeten nach seinen persönlichen und sozialen Verhältnissen zu betreuen, insbesondere ihm einen seiner Leistungsfähigkeit entsprechenden Platz im Erwerbsieben auf jede Weise zu verschaffen, bei seinen Angehörigen und der übrigen Umgebung Verständnis für seine Bedürfnisse zu wecken, ihm die Hilfe der sozialen Fürsorgeeinrichtungen zu vermitteln, sein Selbstvertrauen zu stärken, ihm nach Möglichkeit zur selbständigen Lebensführung zu verhelfen und auf diese Weise sich selbst überflüssig zu machen. Da für den geisteskranken Hilfsbedürftigen eine unmittelbare Therapie nur in sehr beschränktem Umfange in Frage kommt, ist der psychotherapeutischen Wirkung dieser sozialen Fürsorgemaßnahmen, zumal bei der Zugänglichkeit der meisten Kranken für die indirekte suggestive Beeinflussung erhöhte Bedeutung zuzuerkennen. Im Gegensatz zum bisherigen Armenrecht, das sich im Sinne des politischen Liberalismus anf die Gewährung des Existenzminimums beschränkt hatte, enthält das neue Reichsfürsorgerecht (Reichsverordnung über die Fürsorgepflicht vom 13. II. 1924 [RGBl. I, S. 100], sowie Reichsgrundsätze über Voraussetzung, Art und Maß der öffentlichen Fürsorge vom 4. XII. 1924 [RGBl., S. 765]), wenn auch ohne ausdrückliche Nennung der Gesundheitsfürsorge, alle wesentlichen. Grundsätze für eine solche zeitgemäße gesundheitsfïrrsorgerische Hilfeleistung und schafft so die gesetzliche Grundlage für die dem psychotherapeutischen Bedürfnis namentlich hinsichtlich der individuellen Betreuung und der Arbeitspädagogik entsprechenden Maßnahmen der offenen psychiatrischen Fürsorge. Andererseits können die durch das neue Reichsfürsorgerecht vorgeschriebenen Leistungen gegenüber den geisteskranken Hilfsbedürftigen von der öffentlichen Irrenfürsorge nur auf dem Wege der Ergänzung der Anstaltspflege durch die offene Fürsorge in die Wirklichkeit umgesetzt werden.

4. Für alle übrigen Spezialgebiete der offenen Gesundheitsfürsorge wird von den führenden Gesundheitspolitikern ein enges Zusammenarbeiten der offenen Gesundheitsfürsorge und der Krankenhauspflege, ähnlich wie es sich in Nordamerika bewährt hat, auf Grund beweisender gesundheits-wirtschaftlicher Berechnungen und nicht weniger aus Motiven einer auf die Stärkung des Gesundheitswillens gerichteten ärztlichen Psychologie und Pädagogik gefordert und mit Hilfe der "sozialen Krankenhausfürsorge" und entsprechender Arbeitsgemein- 
schaften angestrebt. Dieselben psychotherapeutischen Gesichtspunkte sprechen neben den analogen wirtschaftlichen Erwägungen in erhöhtem Maße für ein enges Handinhandgehen der offenen und der geschlossenen Fürsorgeform innerhalb der Psychiatrie. Der lückenlosen Kontinuität der psychotherapeutischen Führung innerhalb und außerhalb der geschlossenen Anstalt kommt für den Erfolg des Entlassungs- und Wiedereingliederungsversuchs des gebesserten Anstaltskranken wie für die Betreuung des frei lebenden Kranken entscheidende Bedeutung zu. Hieraus erklären sich die epochemachenden Erfolge des Kolbschen Systems der von der Anstalt aus unternommenen und betriebenen offenen Fürsorge, bei der der Hilfsbedürftige vor und nach der Anstaltsentlassung in ein und derselben psychotherapeutischen Hand bleibt ${ }^{1}$. Andererseits hat sich bei der kommunalen Organisation einer selbständigen offenen psychiatrischen Fürsorge stets rasch die Notwendigkeit eines fortlaufenden intimen Zusammenarbeitens mit den zuständigen Irrenanstalten herausgestellt.

5. Die psychotherapeutischen Besonderheiten der offenen Geisteskrankenfürsorge treten beim Vergleich mit der geschlossenen Anstaltsbehandlung deutlich hervor. Seitdem die Rolle des psychogenen Faktors in der Symptomatologie der Psychosen, vor allem dank Bleulers Beobachtungen über Versetzungs- und Entlassungsbesserungen bei Schizophrenen erkannt und die Tatsache einer schädlichen Wirkung, aber auch die Möglichkeit einer psychotherapeutischen Verwertung des Anstaltsmilieus festgestellt ist, muß die Anstaltsbehandlung, die unter der Herrschaft des Dogmas von der Unbeeinflußbarkeit des Krankheitsverlaufes und infolge der allzu großen Dimensionierung der Anstalten häufig zu einer ausschließlichen und keineswegs immer vorteilhaften Anstaltsverwahrung geworden war, unter Wiederaufnahme der früheren irrenärztlichen Bemühungen zu einer planmäßigen Psychotherapie entwickelt werden, wobei weniger die im Gebrauch beschränkte direkte Suggestion als die indirekte Methode der psychischen. Milieuwirkung zu verwerten ist. In dieser Richtung liegt der Erfolg der von Simon aktiver gestalteten Arbeitstherapie, die in ihrer glücklichen Anwendung auf die erregten und unsozialen Kranken eine Neuentdeckung darstellt und als weiterer Beweis für die Suggestibilität der Anstaltsinsassen wie für die massensuggestive Wirkung dieser wie jeder allgemein durchgeführten Behandlungsweise innerhalb der Anstalt anzusprechen ist.

1 Nach dem immer allgemeiner Eingang findenden Erlanger System werden z. B. zur Zeit in Mittelfranken von der Heil- und Pflegeanstalt in Frlangen aus über 2200 Personen, im Freistaat Baden von den Heil- und Pflegeanstalten Illenau, Emmendingen, Wiesl och und bei Konstanz aus über 1700 Personen betreut. 
Im Gegensatz zu diesen massensuggestiven Behandlungsmethoden der Anstalt besteht die offene Geisteskrankenfürsorge wie jede offene Gesundheitsfürsorge in genauem Eingehen auf die persönlichen, familiären und sozialen Verhältnisse des Einzelfalles, in der Zurückführung des gebesserten Kranken auf seinen individuellen Platz im F'amilien- und Erwerbsleben und in der psychotherapeutischen. Auswertung der Wiederaufnahme der produktiven Lohnarbeit, durch die der Kranke seine soziale Rehabilitierung und damit seine Wiederherstellung sich täglich selbst zu beweisen vermag. Die offene Geisteskrankenfürsorge beruht demnach auf der individuellen Ausnützung der Beeinflußbarkeit des einzelnen Kranken. Ist es die Aufgabe der Arbeitstherapie in der Anstalt, den Schizophrenen durch sinnvolle Betätigung vor dem Versinken in den Autismus auf den Abteilungen für Unsoziale zu retten, so ist es die Aufgabe der offenen Fürsorge, ihn durch Wiederanschluß an das Gesellschaftsleben vor dem Versinken in den Beschäftigungsautomatismus innerhalb der Anstalt zu bewahren. Die Arbeitstherapie - auch in ihrer aktivsten Form - kann deshalb nur auf dem Wege der planmäßigen Ergänzung durch die offene Fürsorge vior dem Abgleiten in einen neuen Schematismus, der das wertvolle therapeutische Mittel der sinnvollen Betätigung zum mißverstandenen Selbstzweck werden ließe, geschützt werden. Dabei kann bei den Schizophrenen nur die lange fortgesetzte therapeutische BBemühung, die auch bei schwierigen Fällen vor gewagten Versetzungsund Entlassungsexperimenten nicht zurückschreckt, die endgültig refraktären, der dauernden Anstaltsverpflegung bedürftigen Fälle erweisen.

Auch die Familienpllege, die Unterbringung seitens der Anstalt in einer fremden Familie gegen Entgelt, die mit der offenen Fürsorge die sinnvolle Betätigung außerhalb des Anstaltsbereiches und mit der Arbeitstherapie die Zugehörigkeit zum Anstaltsverband gemein hat, ist ihrer Idee nach nur als Durchgangsetappe ins freie Leben, als therapeutisches Mittel zum Zweck der sozialen Wiedereingliederung zu betrachten, wenn sie auch in ländlichen Bezirken als Vorstufe zur offenen Fürsorge organisiert werden kann.

Die offene Fürsorge ist demnach geeignet, die Maßnahmen der Anstaltsbehandlung, die durch ihren massensuggestiven Charakter in ihrem speziellen therapeutischen Effekt nur allzu leicht beeinträchtigt werden, auf die individuelle Wahrnehmung des einzelnen Kranken zu zentrieren und so ihre Entwicklung zu einer wahren Psychotherapie zu fördern.

6. Hinsichtlich der Psychopathen setzt die von der Anstalt ausgehende offene Fürsorge an die Stelle der stets wiederholten vergeblichen Be- 
einflussungsversuche in der Anstalt erstmals die Möglichkeit, ein planmäßiges psychagogisches Erziehungssystem mit progressivem Charakter unter Beiziehung aller in Betracht kommenden Faktoren und nötigenfalls unter Anwendung der , Schutzaufsicht" vermittels der nachgehenden Überwachung durchzuführen. Hierbei findet die Beeinflussung in der Anstalt an der Aussicht auf die Bewährungsmöglichkeit unter der Kontrolle der offenen Fürsorge eine pädagogisch wertvolle Stïtze und gewinnt andererseits die Beaufsichtigung durch die offene Fürsorge durch die Möglichkeit der sofortigen Zurückverbringung in die Anstalt den unbedingt notwendigen Nachdruck.

Ist die Fürsorge für die Geisteskranken im engeren Sinne dem Fürsorgewesen als besonderer neuer $Z$ weig anzugliedern, so hat sich die Fürsorge für die Psychopathen in der Regel den bisher schon vorhandenen einschlägigen Organisationen, der Trinkerfürsorge, der Fürsorge für jugendliche Psychopathen, der Geführdetenfürsorge, der Gerichtshilfe, der Gefangenenfürsorge und der Fürsorge für Strafentlassene ein zufügen und so die Arbeit dieser Verbände durch die auf die Dauer unentbehrliche fachpsychiatrische Beratung zu fördern.

7. Die offene psychiatrische Fürsorge entspricht durch die Überwachung aller zur Gemeingefährlichkeit neigenden geistig Abnormen ihres Bezirks einem längst geltend gemachten, aber bisher nicht berücksichtigten Bedürfnis des Schutzes der öffentlichen Sicherheit. In Verbindung mit der „Schutzaufsicht“" wird sie künftig bei der Überwachung der Vermindert-Zurechnungsfähigen der kriminellen Prophylaxe wichtige Dienste zu leisten berufen sein.

8. Die offene Geisteskrankenfürsorge bringt die Irrenanstalt in die bisher fehlende engere Berührung mit dem öffentlichen Leben und macht ihre Leistungen der Allgemeinheit bekannt. Sie gliedert die Irrenfürsorge dem allgemeinen Fürsorgewesen ein und verschafft ihr so die bisher mangelnde Anerkennung als unentbehrliches Fachgebiet der sozialen Hygiene. Auf diese Weise dient sie der Aufklärung der Berölkerung über das Wesen des abnormen Seelenlebens und über die Wege zu seiner Verhütung. Bei einer solch bedeutsamen Weiterentwicklung der Irrenpflege wird die individuelle Psychotherapie zur sozialen psychischen Hygiene und zur sozialen psychischen Prophylaxe ${ }^{1}$.

1 Die hier behandelten Fragen finden eine eingehende Darstellung in einer von dem Verfasser in Gemeinschaft mit Kolb und Faltlhauser verfaßten, demnächst bei Julius Springer, Berlin erscheinenden Monographie über „,Die offene Fürsorge in der Psychiatrie und ihren Grenzgebieten". 


\section{Nachtrag zur Generaldiskussion.}

Kehrer-Münster :

Die Einrichtung einer Generaldikussion darf wohl in erster Linie als Aufforderung zu einer kritischen Stellungnahme zum Kongreß als Ganzes gedeutet werden. Eine solche scheint mir im Interesse der weiteren Entwicklung der psychotherapeutischen Kongresse nicht bloß wünschenswert, sondern geradezu geboten. Gerade mit Rücksicht auf die zum Teil weit über dem Durchschnitt stehenden formalen Qualitäten der Referate und Diskussionsbemerkungen zum Thema: Stand der Analyse, ist es erforderlich, gegen das dabei zutage getretene Überwuchern rein theoretischer, spekulativer, philosophistischer Betrachtungsweisen Stellung zu nehmen. Insbesondere habe ich das A und das $\mathrm{O}$ vermißt: einmal die Darstellung der Ursachen und Bedingungen, welche beim Walten Freudscher Mechanismen, die ja fast in uns allen tätig sind oder sein sollen, von Fall zu Fall bestimmen, ob es zu „Hysterie“, Angstzuständen, Zwangsleiden, Sexualabnormität usw. und dabei zu recht versehiedenen Verläufen dieser kommt; zum anderen die vorur. teilsfreie, sachliche Berichterstattung über die ,psychoanalytisehen“ Behandlungserfolge bei diesen verschiedenen Zuständen, entsprechend dem sonst in der Medizin mit Recht herrschenden Brauche. Nur so vermag man zu erkennen, wieweit die psychoanalytischen Deutungen Anspruch auf Allgemeingültigkeit erheben können, d. h. von jedem vorurteilslos denkenden und fühlenden Wissenschaftler als richtig anerkannt werden müssen und wieweit ferner die psychoanalytische Behandlungsmethode wirklich für alle Kreise psychotherapeutischer Klientel geeignet und möglich ist. Was die Mehrzahl der führenden Psychiater und Neurologen an den Universitäten und viele Ärzte sonst noch immer gegen die Psychotherapie, aber ganz besonders die Psychoanalyse mißtrauisch macht, ist wohl in erster Linie dieser hier in die Erscheinung getretene Mangel an erfahrungswissenschaftlicher Nüchternheit und Klarheit. Den Satz: gleiche Ursachen und gleiche Bedingungen, gleiche Symptome, gleiche Verläufe und evtl. auch gleiche Behandlungserfolge bat sich die Psychoanalyse noch nicht zu eigen gegemacht. Es dünkt mir besonders symptomatisch, daß man sehr lebhaft sich darüber gestritten hat, ob Psychoanalyse Natur-oder Kultur-oder was sonst für eine Wissenschaft sei und dabei ganz übersah, daß es m. E. einzig und allein darauf ankommt, daß sie als Psychotherapie erfahrungswissenschaftlich gesicherte Methodik psychischer Mittel ist. Die Theorie der Psychoanalyse, wie wir sie gehört haben, hat dabei streng genommen mit Psychotherapie ja nichts zu tun, denn sie ist psychologische Theorie im Grunde wie jede andere. Mir wäre es daher auch richtiger erschienen, auf diesem Kongreß nicht bloß die Freudsche, 
284 Bericht über den 2. allgemeinen ärztlichen Kongreß für Psychoterapie.

sondern alle ihr verwandten ,Psychologien" gemeinsam unter den von mir bezeichneten erfahrungswissenschaftlichen Gesichtspunkten zum Gegenstand der Verhandlung zu machen. Nur die dauernde und nachdrückliche Besinnung auf die Erfordernisse der psychotherapeutischen Praxis im weitesten Sinne gibt unseren Kongressen Existenzberechtigung und läßt sie gedeihen.

Eigenberichte (durch Kankeleit-Hamburg). 